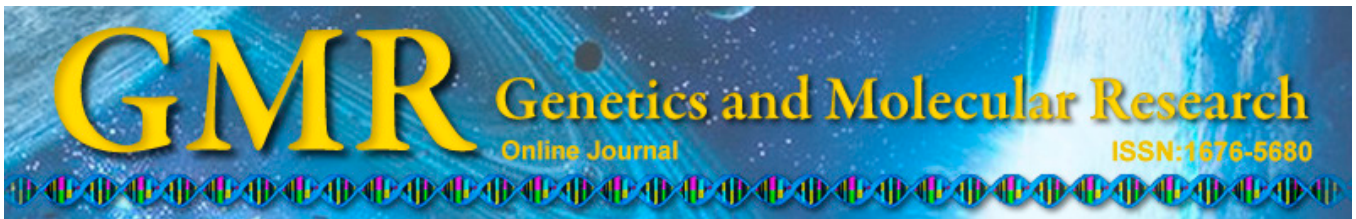

\title{
Evolutionary analysis of the ubiquitin gene of baculovirus and insect hosts
}

\author{
S.S. Ma, Z. Zhang, H.C. Xia, L. Chen, Y.H. Yang, Q. Yao and K.P. Chen \\ Institute of Life Sciences, Jiangsu University, Zhenjiang, Jiangsu, China \\ Corresponding author: K.P. Chen \\ E-mail: kpchen@ujs.edu.cn
}

Genet. Mol. Res. 14 (3): 9963-9973 (2015)

Received January 27, 2015

Accepted May 17, 2015

Published August 21, 2015

DOI http://dx.doi.org/10.4238/2015.August.21.2

\begin{abstract}
Baculovirus is the only virus that has been found to encode the ubiquitin protein. In this study, ubiquitin sequences from 16 insects and 49 viruses were collected and compared. The resulting sequences were aligned with virus genomes. Then MAGE 5.0, k-estimated software, as well as other software programs were used for systemic evolutionary, selection pressure, and evolutionary distance analysis. The results of the pairwise ratio of non-synonymous to synonymous substitution values and evolutionary distances showed that ubiquitin from baculovirus and insect hosts have been under purifying selection during evolution and are thus evolutionarily conserved. Moreover, genes from insect hosts were more conserved than those in baculovirus. Analysis of the non-synonymous to synonymous substitution rates at each site and entropy calculations revealed the evolutionary status of every site in the ubiquitin genes of baculovirus and their hosts. Genome locations and phylogenetic trees indicated that granuloviruses and non-photosynthetic vegetation evolved, and granulovirus evolution was more similar to that of insect hosts. Our results suggest that the ubiquitin gene in baculovirus may have been acquired through horizontal transfer from the host.
\end{abstract}

Key words: Baculovirus; Conservation; Evolution; Insect hosts; Ubiquitin 


\section{INTRODUCTION}

Baculovirus exists in insects and parts of crustacean arthropods. Baculoviruses are divided into 2 genera, nuclear polyhedrosis virus (NPV) and granulovirus (GV), and include more than 600 species (Theilmann et al., 2005). Baculovirus is the only virus that has been shown to encode the ubiquitin protein. All sequenced baculoviruses that have been tested encode ubiquitin protein except Leucania separata NPV, Culex nigripalpus NPV, Neodiprion abietis NPV, Neodiprion lecontei NPV, and Neodiprion sertifer NPV.

Ubiquitin is highly conserved in eukaryotes and has similar functions and immunological effects. Ubiquitin degrades nuclear and cytoplasmic proteins effectively and specifically mainly through the ATP-dependent ubiquitin-proteasome pathway. In addition, as a molecular chaperone, ubiquitin is involved in many other physiological functions. Ubiquitin from baculovirus is anchored in the membrane of budding virus particles by binding with phospholipids through covalent bonding (Guarino et al., 1995). A study of Autographa californica NPV revealed that the ubiquitin gene has no direct relationship with replication of the virus genome, but the absence of the ubiquitin gene decreases baculovirus particle production by 5-10 times (Relly and Guarino, 1996). In another study, deletion of viral ubiquitin from the Bombyx mori NPV (BmNPV) genome did not affect baculovirus and occlusion body production in BmNPV-infected BmN cells, differing from A. californica NPV (Katsuma et al., 2011). In addition, the viral ubiquitin molecule surface does not possess the locus Lys48 identification signal for the formation of polyubiquitin chains, but the ubiquitin of baculovirus may also be involved in the ubiquitin-proteasome pathway, which is similar to eukaryotic ubiquitin protein (Haas et al., 1996).

Long-term interactions between viruses and hosts lead to co-evolution. These two compete, mutually selecting and adapting with each other during the evolutionary process. For example, polyhedrin of BmNPV can release virus particles for launch infection only under the larval midgut alkaline $\mathrm{pH}$ condition. The alkaline environment in the insect host intestine and the dissolution of virus polyhedrin crystals under alkaline conditions are the result of longterm evolution of hosts and viruses. At the molecular level, the evolution of virus proteins shows the same pattern. In previous studies, various baculovirus genes were used as the basis of viral evolution, such as lef-2 (Chen et al., 1999), egt (Chen et al., 1997), DNA polymerase (Bulach et al., 1999; Nielsen et al., 2002), chiA, lef-8, ac22 (Kang et al., 1998), gp41 (Liu and Maruniak, 1999), and polh (Rohrmann, 1986; Zanotto et al., 1993). Interestingly, some proteins in baculovirus exhibit horizontal transfer, including horizontal communication between the host and virus gene. Hughes and Friedman (2003) detected this transfer between host cells and baculovirus using 13 different baculovirus genomes, and found that 6 genes in baculovirus indicated horizontal transfer of the ubiquitin gene. Among these 6 genes, the ancestors of UDP glucuronosyltransferase, inhibitor of apoptosis 1, and Chi originated in the hosts (Hairong, 2011), but there is no conclusive evidence to indicate the source of the ubiquitin gene.

In viruses, the existence of ubiquitin in baculovirus is a unique feature. Whether its presence is associated with host evolution or its own evolution is currently unclear. In this study, ubiquitin sequences from 16 insects and 49 viruses were identified and compared. In addition, the evolution and classification of ubiquitin genes in baculovirus and hosts were subjected to bioinformatic analysis to analyze the evolution of the system, selection pressure, and evolutionary distance. 


\section{MATERIAL AND METHODS}

\section{Sequence collection and location of ubiquitin in baculovirus genomes}

Ubiquitin cDNA sequences and amino acid sequences of B. mori and BmNPV were used as reference sequences and were searched in the silkbase database and National Center for Biotechnology Information (NCBI) database using the BLASTn and TblastN tools. Sequences were selected when the e value $<10^{-5}$ and the coverage rate was more than $90 \%$ as candidate sequences; 49 viruses were identified. Ubiquitin from 16 insects were identified manually, including 8 insects containing a corresponding virus and 8 other insects. In the NCBI genome database, ubiquitin localization was determined by analyzing 3 up- and 3 downstream genes of the ubiquitin gene.

\section{Sequence alignment and phylogenetic reconstruction}

All virus and host ubiquitin gene cDNA sequences were compared using the Muscle algorithm in the MEGA5 software (Tamura et al., 2011). Using the MEME online tool (Bailey et al., 2009), amino acid-level multiple sequence alignment was conducted for 49 baculoviruses and 16 insect host ubiquitin sequences. The maximum likelihood (ML) algorithm in MEGA5 was used for phylogenetic tree reconstruction of all sequences, the bootstrap value was set to 1000, and the optimal model $\mathrm{K} 2+\mathrm{I}+\mathrm{G}$ was calculated using the JmodelTest. PAUP and phyML were used to construct the neighbor joining and ML trees, respectively, within bootstrap values of 1000 and a best fit model of $\mathrm{K} 2+\mathrm{I}+\mathrm{G}$. Both trees were reconstructed to supplement the phylogenetic tree reconstructed using MEGA5.

\section{Evolutionary distance and positive selection}

The 65 sequences were grouped into the virus ubiquitin group ( 49 sequences) and host ubiquitin group (16 sequences). MEGA5 was used to calculate the evolutionary distance within and between the 2 groups. Within each group, the ratio of non-synonymous to synonymous substitution $(\mathrm{dN} / \mathrm{dS})$ values of every 2 sequences of all the sequences were calculated and drawn in a dN/dS scatter diagram. The HYPHY algorithm was used to calculate selection pressure at each site of all sequences within each group, obtain the $\mathrm{dN} / \mathrm{dS}$ value, and draw the $\mathrm{dN} / \mathrm{dS}$ curve. The k-estimated software was used to analyze the sliding-window $\mathrm{dN} / \mathrm{dS}$ values for both virus and host ubiquitin proteins. The window was 90 base pairs (bp) and the step was $30 \mathrm{bp}$.

The Boltzmann-Shannon entropy $\mathrm{H}$ value was used to quantify the sequence variability of amino acid residues at each site and was calculated as

$$
H(P)=-\sum_{j=1}^{20} p_{j} \log _{20}\left(p_{j}\right)
$$

where $p_{j}$ is the probability of an amino acid being of the $\mathrm{j}^{\text {th }}$ kind, and $0 \leq \mathrm{H}(\mathrm{P}) \leq 1$ (Atchley and Fernandes, 2005).

\section{RESULTS}

\section{Gene search and linear relationship}

Sixty-five ubiquitin gene sequences from 16 hosts and 49 viruses were searched (Table 1), of which the 16 hosts of virus had corresponding accession numbers in GenBank; the other 34 were not found. We collected 3 upstream and 3 downstream genes to investigate the 
synteny of ubiquitin genes in the viral genome. According to the difference between upstream and downstream sequences of the ubiquitin gene, 49 types of baculovirus could be divided into 2 categories, including the GV group with the DUF1443 and DUF816 genes upstream and the DUF673 and 39K genes downstream; the other group was NPV, which included the DUF1247 and 39K genes and the LEF-11 DBP genes upstream and downstream, respectively (Figure 1). This suggests that the location of ubiquitin in the GV and NPV genus are different, and implies that the 2 genus viruses may have undergone different evolutionary processes.

\begin{tabular}{|c|c|c|c|c|}
\hline Virus & GenBank ID & Location & Hosts & GenBank ID \\
\hline AgseGV & NC_005839 & $48,433-48,726$ & & \\
\hline CrleGV & NC_004968 & $43,891-44,121$ & & \\
\hline AdorGV & NC_004938 & $33,085-33,415$ & & \\
\hline PhopGV & NC_004062 & $42,825-43,109$ & & \\
\hline $\mathrm{HaGV}$ & $\mathrm{NC}_{-}^{-} 010240$ & $41,975-42,208$ & Helicoverpa armigera & AY456195 \\
\hline SpliGV & NC_009493 & $33,846-34,127$ & Spodoptera litura & AF436065 \\
\hline ChocGV & NC_008168 & $38,030-38,317$ & & \\
\hline XcGV & $\mathrm{NC}_{-}^{-} 002331$ & $42,949-43,183$ & & \\
\hline CypoGV & $\mathrm{NC}_{-}^{-} 002816$ & $44,952-45,336$ & & \\
\hline PlxyGV & $\mathrm{NC}_{-}^{-} 002593$ & $34,108-34,452$ & Plutella xylostella & EU428781 \\
\hline PiraGV & NC_013797 & $39,432-39,719$ & Pieris rapae & DQ780001 \\
\hline PsunGV & NC_013772 & $46,360-46,593$ & & \\
\hline CfGV & AY $0 \overline{4} 8769$ & & & \\
\hline AcMNPV & NC_001623 & $28,962-29,195$ & & \\
\hline BomaNPV & NC_012672 & $25,189-25,422$ & Bombyx mandarina & DQ839401 \\
\hline CfDefMNPV & NC_005137 & $19,975-20,208$ & & \\
\hline CfMNPV & NC_004778 & $18,805-19,089$ & & \\
\hline RoMNPV & NC_004323 & $27,109-27,342$ & & \\
\hline AgMNPV & $\mathrm{NC}_{-}^{-} 008520$ & $21,555-21,791$ & & \\
\hline EppoNPV & NC_003083 & $18,095-18,325$ & & \\
\hline OpMNPV & NC_001875 & $20,894-21,175$ & & \\
\hline BmNPV & NC_001962 & $24,935-25,268$ & Bombyx mori & AF308163 \\
\hline HycuNPV & $\mathrm{NC}_{-}^{-} 007767$ & $115,286-115,525$ & & \\
\hline AnpeNPV & $\mathrm{NC}_{-}^{-} 008035$ & $16,705-16,935$ & & \\
\hline MaviNPV & $\mathrm{NC}^{-} 008725$ & $21,834-22,067$ & & \\
\hline PlxyMNPV & NC_-008349 & $30,232-30,465$ & Plutella xylostella & EU428781 \\
\hline AnpeMNPV & EF_ 207986 & $109,929-110,159$ & & \\
\hline ClbiNPV & NC_008293 & $24,999-25,249$ & & \\
\hline TnSNPV & $\mathrm{NC}^{-} 007383$ & $23,991-24,224$ & Trichoplusia ni & AY267009 \\
\hline ChchNPV & $\mathrm{NC}_{-}^{-} 007151$ & $26,769-27,005$ & & \\
\hline HaMNPV & NC_011615 & $138,600-138,902$ & Helicoverpa armigera & AY456195 \\
\hline MacoNPV B & NC_004117 & $142,882-143,184$ & & \\
\hline HzSNPV & NC_-003349 & $24,652-24,903$ & & \\
\hline SpltNPV & NC_003102 & $31,136-32,191$ & Spodoptera litura & AF436065 \\
\hline HaNPV C1 & NC_003094 & $24,832-24,983$ & Helicoverpa armigera & AY456195 \\
\hline SeMNPV & NC_002169 & $118,496-118,738$ & Spodoptera exigua & AY149883 \\
\hline LdMNPV & $\mathrm{NC}_{-}^{-} 001973$ & $40,690-41,142$ & & \\
\hline EupsNPV & $\mathrm{NC}_{-}^{-} 012639$ & $45,104-45,340$ & & \\
\hline HaNPV NNg1 & NC_011354 & $24,897-25,148$ & Helicoverpa armigera & AY456195 \\
\hline AiMNPV & $\mathrm{NC}^{-} 011345$ & $138,365-138,614$ & & \\
\hline OrleNPV & $\mathrm{NC}^{-} 010276$ & $64,192-64,443$ & & \\
\hline EcobNPV & $\mathrm{NC}^{-} 008586$ & $22,219-22,458$ & & \\
\hline AdhoNPV & NC_004690 & $15,121-15,363$ & & \\
\hline SfMNPV & NC_009011 & $114,140-114,382$ & Spodoptera frugiperda & JX087454 \\
\hline MacoNPV A & $\mathrm{NC}_{-}^{-} 003529$ & $139,462-139,764$ & & \\
\hline HaNPV G4 & $\mathrm{NC}_{-}^{-} 002654$ & $24,925-25,176$ & Helicoverpa armigera & AY456195 \\
\hline SpltNPV II & NC_-011616 & $126,877-127,113$ & Spodoptera litura & AF436065 \\
\hline AdorNPV & $\mathrm{NC}_{-} 011423$ & $14,934-15,176$ & & \\
\hline AgseNPV & NC_007921 & $130,998-131,234$ & & \\
\hline LXMNPV & NC_013953 & $38,244-38,699$ & & \\
\hline
\end{tabular}




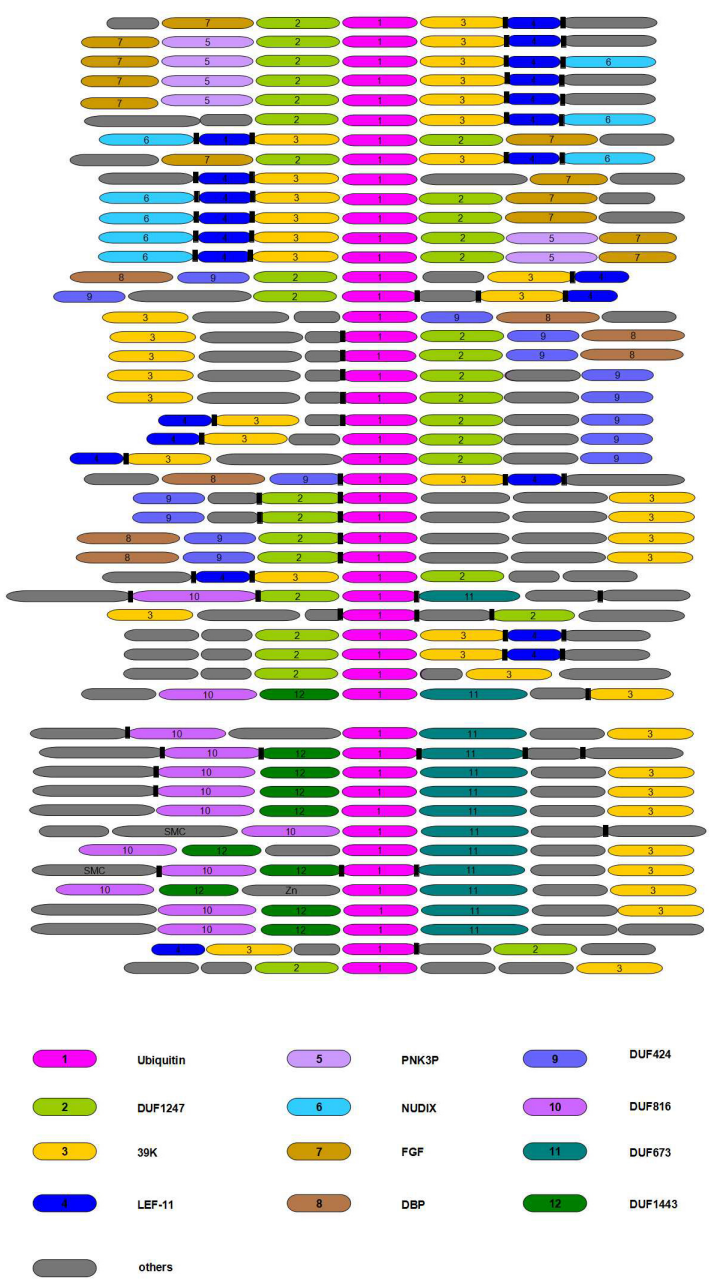

Figure 1. Linear relationship between ubiquitin proteins in both viruses and hosts. Linear relationship between ubiquitin proteins in the virus genome. Different colors indicate different genes. The black box indicates that the 2 sequences overlap.

\section{Motif analyses and Boltzmann-Shannon entropy}

Using the muscle method in MEGA5 for amino acid-level multiple sequences, we aligned 49 baculovirus and 16 insect host ubiquitin sequences. At the amino acid level, ubiquitin sequences of virus and insect hosts were conserved, and only differed in some sites, while the ubiquitin sequences of insect hosts were more conserved. MEME online analysis predicted that virus ubiquitin has 2 motifs (Figure 2A); motif 1 corresponded to amino acid sites 1-20 in the ubiquitin sequence, while motif 2 corresponded to amino acid sites 26-75. Insect host ubiquitin sequences were predicted to possess 3 motifs (Figure 2B), with motif 1 corresponding to amino acid sites 1-15 in the ubiquitin sequence, motif 2 corresponding to amino acid sites 19-68, and motif 3 corresponding to amino acid sites 69-76. 

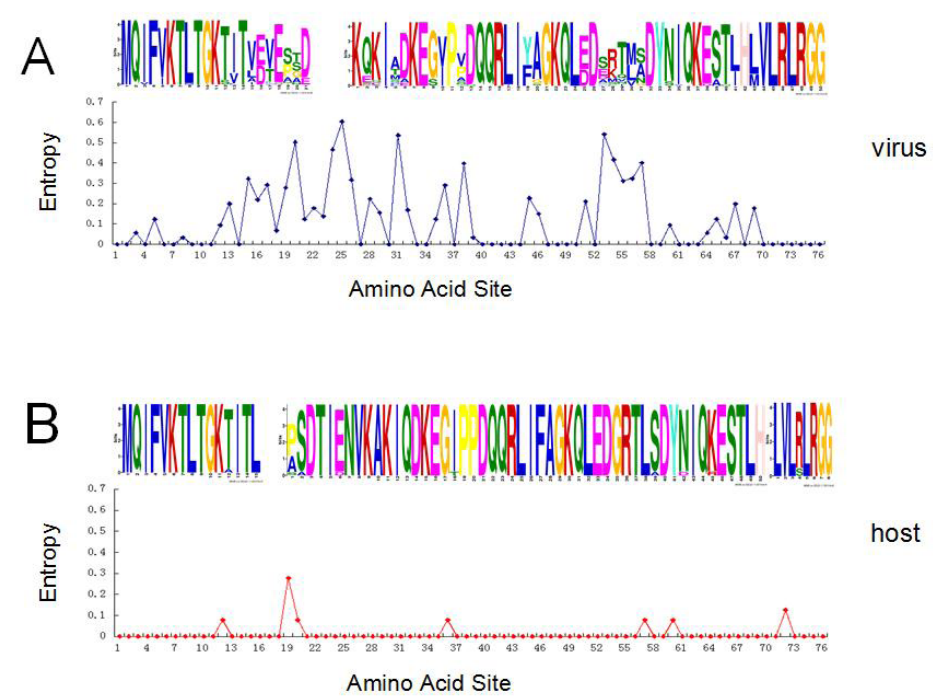

Figure 2. Motif analyses by MEME online tool and line chart of Boltzmann-Shannon entropy both of virus and host. A. motif analyses by MEME online tool and line chart of Boltzmann-Shannon entropy of virus. B. Motif analyses by MEME online tool and line chart of Boltzmann-Shannon entropy of host.

The Boltzmann-Shannon entropy was calculated using equation (1), as the entropy was between 0 and 1 . At each site, a lower value indicated that evolution was more conserved. At most sites, entropy was very low, particularly in the host group (Figure 2). This suggests that evolution of the ubiquitin gene was conservative.

\section{Phylogenetic tree}

A phylogenetic tree was reconstructed with the ML method using all ubiquitin sequences obtained from baculovirus and insects. The results showed that baculovirus and host ubiquitin genes gathered into a cluster. Interestingly, in terms of baculovirus, all GV ubiquitin genes clustered together, while NPVs were divided into 2 groups: the OrleNPV and EcobNPV cluster, which were named as NPV II and stood apart from the other NPV clusters (NPV I). Genes form NPV II suggested that the 2 viruses play an important role in the evolution of the ubiquitin gene (Figure 3). The ML tree (reconstructed using phyml) (Figure S1) and neighborjoining tree (using PAUP) (Figure S2) were consistent with this phylogenetic relationship.

\section{Pairwise dN/dS values in different groups}

Within each group, the $\mathrm{dN} / \mathrm{dS}$ value of every 2 sequences of all the sequences was calculated, and a dN/dS scatter diagram (Figure 4) was drawn. The results showed that the $\mathrm{dN} / \mathrm{dS}$ value of both baculovirus and insect host ubiquitin genes were much lower than 1 , suggesting that ubiquitin genes, both in viruses and in hosts, underwent negative selection. In addition, the $\mathrm{dN} / \mathrm{dS}$ value of host was less than that of the virus, implying that the ubiquitin genes in hosts experienced stronger selection pressure than those in the virus. Thus, ubiquitin from baculovirus and insect hosts were subject to the effects of purifying selection pressure in 
evolution and thus evolved conservatively; the genes of insect hosts were more conservative than those of baculovirus during evolution.

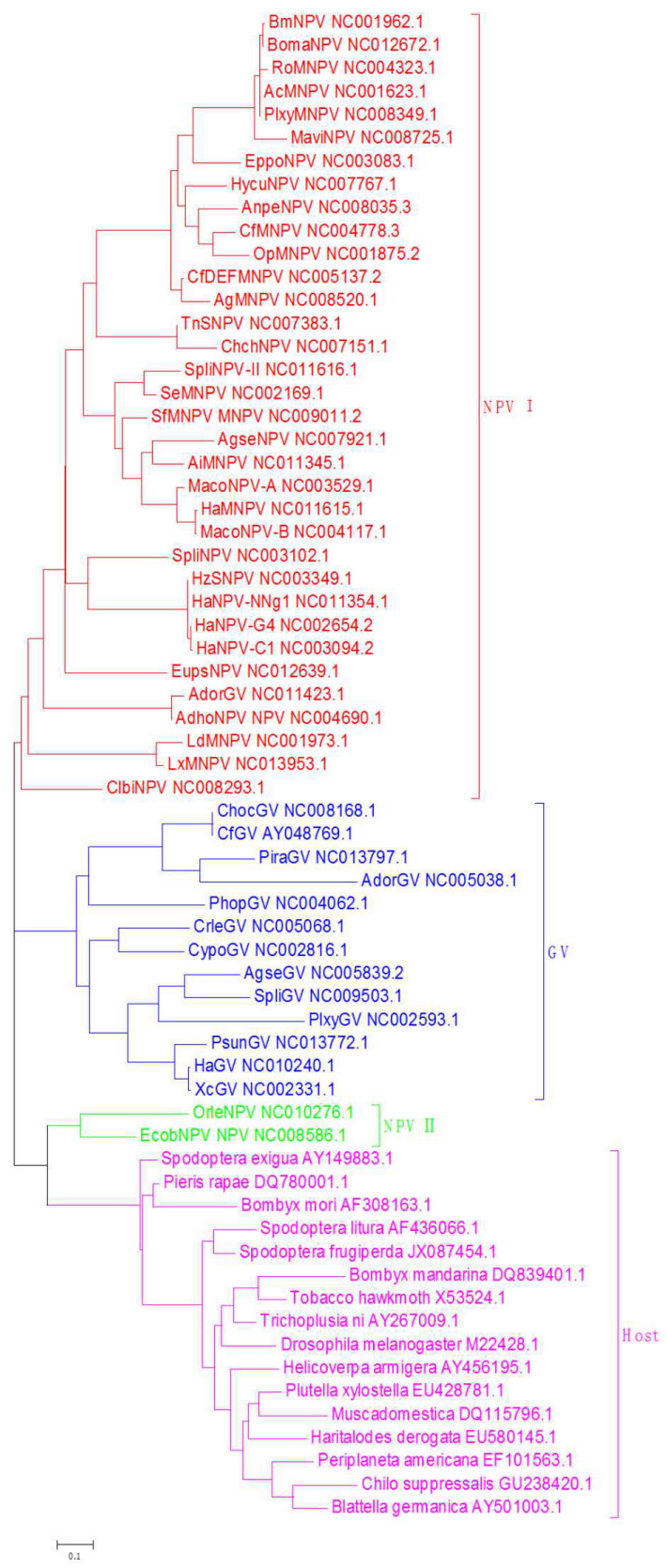

Figure 3. Phylogenetic tree of ubiquitin. Purple, green, blue, and red indicate host, NPV II, GV, and NPV I clades, respectively. The bootstrap value was set to 1000 , and the optimal model K2 + I + G was calculated using JmodelTest. 


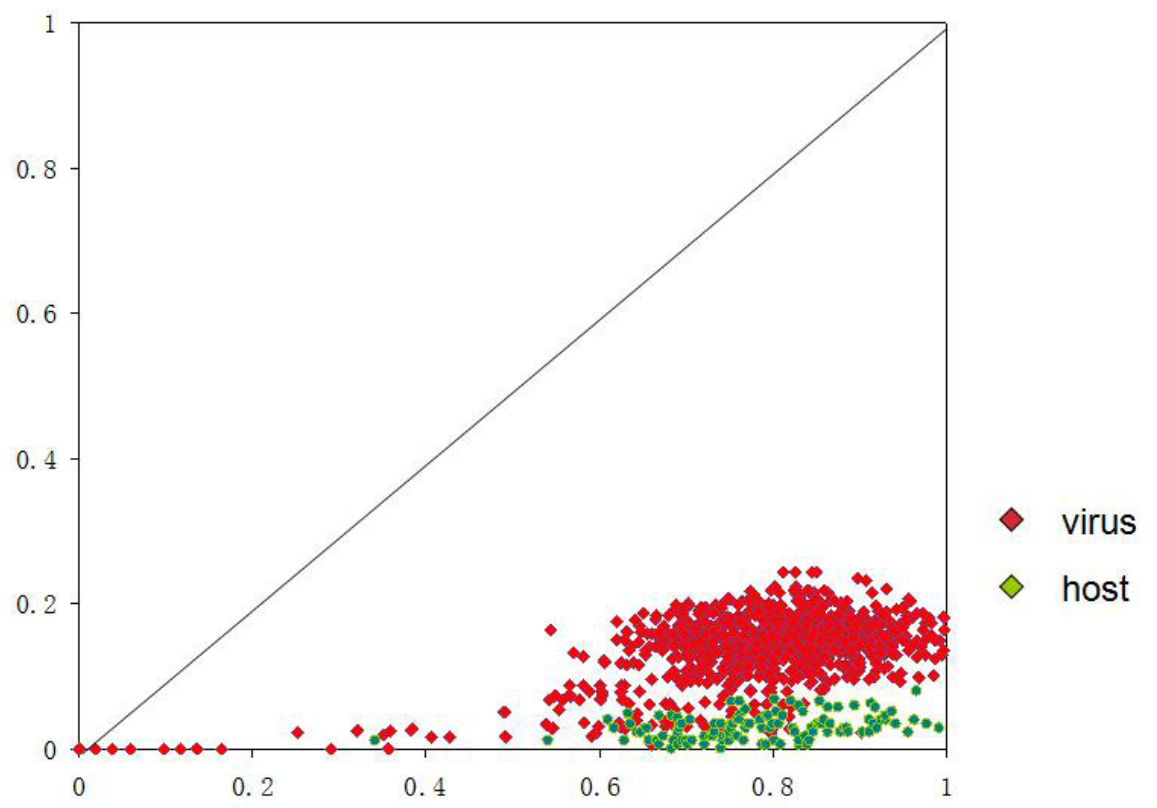

Figure 4. Pairwise dN/dS value of hosts and viruses ubiquitin proteins. dN/dS value of every 2 sequences of all the sequences was calculated using MEGA. Red dots represent virus and green dots represent hosts.

\section{Synonymous and non-synonymous substitutions at each site in the ubiquitin sequence}

The HYPHY algorithm was used to calculate the selection pressure at each site of all sequences within each group to determine $\mathrm{dN} / \mathrm{dS}$ and draw the $\mathrm{dN} / \mathrm{dS}$ curve (Figure 5). The results showed that $\mathrm{dN} / \mathrm{dS}$ values at each site in ubiquitin sequences of both baculovirus and

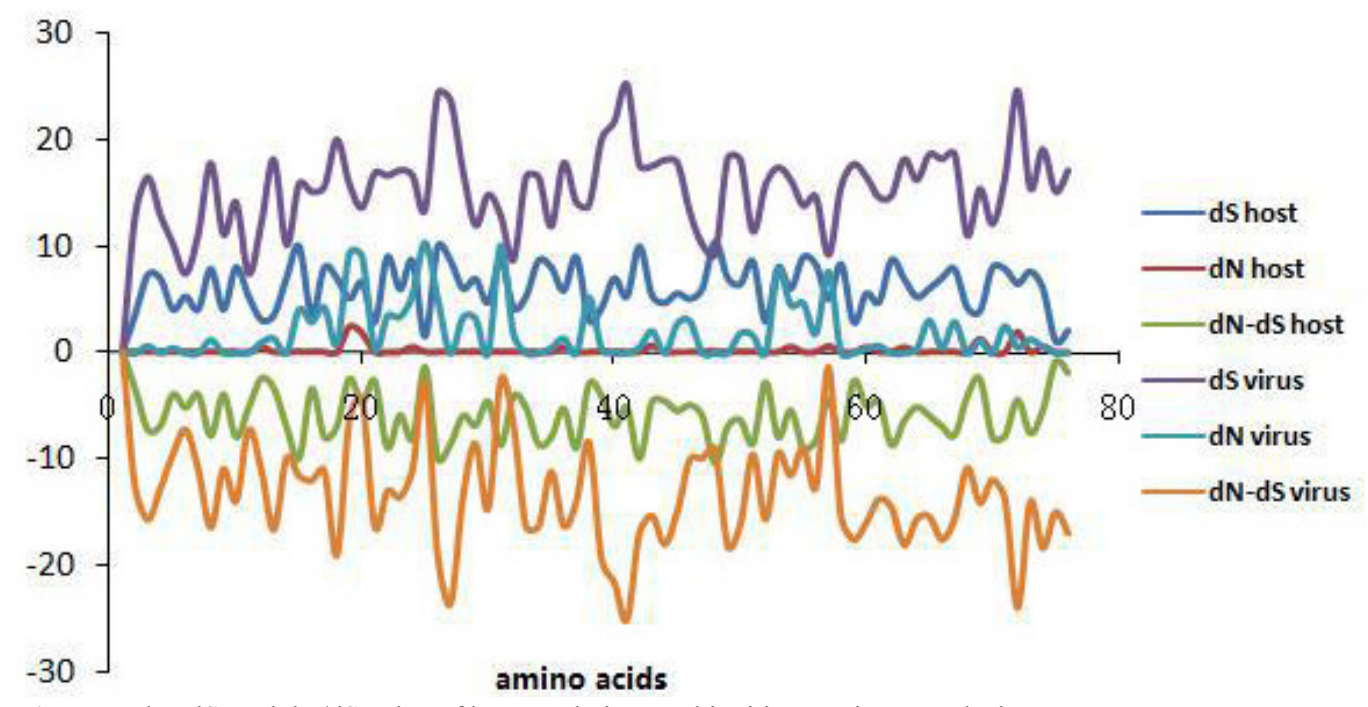

Figure 5. dN, dS, and dN/dS value of hosts and viruses ubiquitin proteins at each site. 
insect hosts were less than 0 , indicating that each site of virus and host ubiquitin genes was under purifying selection effects, and at most sites, purifying selection to the hosts were greater than those to the virus. Thus, host sequences were more conserved at these sites. However, the opposite trend was observed in some cases, such as at site No. $24(\mathrm{dN} / \mathrm{dS}$ host $=-16.581$; $\mathrm{dN} / \mathrm{dS}$ virus $=-13.563)$, site No. $29(\mathrm{dN} / \mathrm{dS}$ host $=-14.078 ; \mathrm{dN} / \mathrm{dS}$ virus $=4.065)$, site No. 48 $(\mathrm{dN} / \mathrm{dS}$ host $=-13.368 ; \mathrm{dN} / \mathrm{dS}$ virus $=-13.247)$, and site No. $57(\mathrm{dN} / \mathrm{dS}$ host $=-4.421 ; \mathrm{dN} / \mathrm{dS}$ virus $=-3.257$ ) (data not shown). Interestingly, amino acid No. 48 is an important active site in the eukaryotic ubiquitin protein. Because the host and virus compete, mutually selecting and adapting with each other in the evolutionary process, these different sites may result from their interaction. Sliding window analysis showed inconsistent results (igure S3 $)$.

\section{Evolutionary distance within groups and between groups}

The distance function in MEGA5 was used to calculate the evolutionary distance within and between the 2 groups (Table 2). The results showed that the evolutionary distance in the baculovirus group was $0.153 \pm 0.023$, which was greater than the evolutionary distance in the insect host group of $0.029 \pm 0.007$; however, the evolutionary distance of the baculovirus and insect host group was $0.169 \pm 0.028$, which is larger than the previous values. This suggested that the ubiquitin gene of baculovirus is more conserved than that in insect hosts during evolution, while the evolutionary rates between virus and host differed.

Table 2. Evolutionary distance within and between groups.

\begin{tabular}{lc}
\hline & Distance \\
\hline Virus within group & $0.153 \pm 0.023$ \\
Host within group & $0.029 \pm 0.007$ \\
Between host and virus groups & $0.169 \pm 0.028$ \\
All sequences & $0.149 \pm 0.024$ \\
\hline
\end{tabular}

\section{DISCUSSION}

Ubiquitin is a small and ubiquitous protein in eukaryotes, but baculovirus is the only virus encoding for the ubiquitin protein. In this study, ubiquitin sequences from 16 insects and 49 viruses were identified and analyzed using evolutionary analysis methods. The results of selection pressure and evolutionary distance analyses showed that ubiquitin of baculovirus and insect hosts were subject to purifying selection pressure in evolution, evolved conservatively, and the genes of insect hosts were more affected than baculovirus during evolution. At some sites, the virus evolutionary rate was lower than that in the insect host, which may have resulted from an interaction between viruses and hosts. Notably, the virus was more affected by purifying selection pressure than hosts at the site Lys48, which is an important active site in eukaryotes. Based on the phylogenetic tree, virus and hosts appear to have evolved together, while NPV and GV evolved independently. Interestingly, the 2 viruses OrleNPV NVP and EcobNPV were not clustered together with the others, but formed a separate cluster, implying that these NPV viruses may have evolved independently and had a very close relationship with their hosts.

The phylogenetic tree of ubiquitin from this study was compared with other evolutionary trees from previous studies such as GP37 (Phanis et al., 1999), UDP glucuronosyltransferase, Chi, inhibitor of apoptosis (Hairong, 2011), alk-exo (Keeling and Palmer, 2008), and 
DNA polymerase (Herniou et al., 2003). According to the position of the ubiquitin gene in baculovirus, 3 upstream and 3 downstream sequences were found. The upstream and downstream sequences from GV and NPV were different. For the GV genus, the upstream sequences of the ubiquitin gene mainly contained DUF1443 and DUF816, while the downstream sequences included DUF673 and 39K. For the NPV genus, the upstream sequences of the ubiquitin gene mainly contained DUF1247 and 39K, while the downstream sequences included LEF-11 and DBP. This shows that GV and NPV differed in their evolutionary processes. In addition, the results of phylogenetic analysis and the location in the genome indicated a relationship: the species that clustered together in the genome location are similar, with similar upstream and downstream sequences. Closer clustering indicates a more similar location.

In selection pressure analyses, there was no significant difference between ubiquitin from baculovirus and hosts, but ubiquitin protein from hosts showed stronger purifying selection pressure than baculovirus. Thus, similar to other proteins, the evolutionary rate of ubiquitin protein in the virus was faster than that in hosts after it was delivered. Positive selection and entropy calculations indicate that each site of virus and host ubiquitin genes was under purifying selection effects, resulting in conservative evolution.

It has been reported that some baculovirus genes may have been derived from the host, such as inhibitor of apoptosis (Huang et al., 2000; Hughes, 2002) and egt (O'Reilly and Miller, 1989). Alk-exo gene clustering suggested that baculovirus clustered according to the different orders of insects. This demonstrates the existence of a co-evolutionary relationship between the virus and its host; similar results have been obtained from cluster analysis of the lef- 8 gene and AC22 gene studied by Herniou et al. (2004). Because the ubiquitin gene of baculovirus and hosts have very high homology, the evolutionary distance between the ubiquitin gene in the virus and insect hosts was not clear, both between and within groups. Phylogenetic analysis also showed that they may have a common ancient ancestor. Thus, we speculate that the ubiquitin gene from baculovirus may have undergone horizontal transfer from the host, similarly to the Chi and UDP glucuronosyltransferase proteins. In this study, we verified the prediction that the ubiquitin gene of baculovirus may have undergone horizontal transfer from the host.

\section{ACKNOWLEDGMENTS}

Research supported by the National Basic Research Program of China under grant \#2012CB114604, the National Natural Science Foundation of China under grant \#31372259, and the Postdoctoral Science Foundation of China under grant \#2013M531289.

\section{Supplementary Material}

\section{REFERENCES}

Atchley WR and Fernandes AD (2005). Sequence signatures and the probabilistic identification of proteins in the MycMax-Mad network. Proc. Natl. Acad. Sci. U. S. A. 102: 6401-6406.

Bailey TL, Boden M, Buske FA, Frith M, et al. (2009). MEME SUITE: tools for motif discovery and searching. Nucleic Acids Res. 37: W202-W208.

Bulach DM, Kumar CA, Zaia A, Liang B, et al. (1999). Group II nucleopolyhedrovirus subgroups revealed by phylogenetic analysis of polyhedrin and DNA polymerase gene sequences. J. Invertebr. Pathol. 73: 59-73.

Chen X, Hu Z, Jehle JA, Zhang Y, et al. (1997). Analysis of the ecdysteroid UDP-glucosyltransferase gene of Heliothis 
armigera single-nucleocapsid baculovirus. Virus Genes 15: 219-225.

Chen X, IJkel WF, Dominy C, de Andrade Zanotto PM, et al. (1999). Identification, sequence analysis and phylogeny of the lef-2 gene of Helicoverpa armigera single-nucleocapsid baculovirus. Virus Res. 65: 21-32.

Guarino LA, Smith G and Dong W (1995). Ubiquitin is attached to membranes of baculovirus particles by a novel type of phospholipid anchor. Cell 80: 301-309.

Haas AL, Katzung DJ, Reback PM and Guarino LA (1996). Functional characterization of the ubiquitin variant encoded by the baculovirus Autographa californica. Biochemistry 35: 5385-5394.

Hairong D (2011). Analysis of horizontal transfer gene of Bombyx mori NPV. Yi Chuan. 33: 636-647.

Herniou EA, Olszewski JA, Cory JS and O'Reilly DR (2003). The genome sequence and evolution of baculoviruses. Annu. Rev. Entomol. 48: 211-234.

Herniou EA, Olszewski JA, O'Reilly DR and Cory JS (2004). Ancient coevolution of baculoviruses and their insect hosts. J. Virol. 78: 3244-3251.

Huang Q, Deveraux QL, Maeda S, Salvesen GS, et al. (2000). Evolutionary conservation of apoptosis mechanisms: lepidopteran and baculoviral inhibitor of apoptosis proteins are inhibitors of mammalian caspase-9. Proc. Natl. Acad. Sci. U. S. A. 97: 1427-1432.

Hughes AL (2002). Evolution of inhibitors of apoptosis in baculoviruses and their insect hosts. Infect. Genet. Evol. 2: 3-10.

Hughes AL and Friedman R (2003). Genome-wide survey for genes horizontally transferred from cellular organisms to baculoviruses. Mol. Biol. Evol. 20: 979-987.

Kang W, Tristem M, Maeda S, Crook NE, et al. (1998). Identification and characterization of the Cydia pomonella granulovirus cathepsin and chitinase genes. J. Gen. Virol. 79: 2283-2292.

Katsuma S, Tsuchida A, Matsuda-Imai N, Kang W, et al. (2011). Role of the ubiquitin-proteasome system in Bombyx mori nucleopolyhedrovirus infection. J. Gen. Virol. 92: 699-705.

Keeling PJ and Palmer JD (2008). Horizontal gene transfer in eukaryotic evolution. Nat. Rev. Gen. 9: 605-618.

Liu JC and Maruniak JE (1999). Molecular characterization of genes in the GP41 region of baculoviruses and phylogenetic analysis based upon GP41 and polyhedrin genes. Virus Res. 64: 187-196.

Nielsen CB, Cooper D, Short SM, Myers JH, et al. (2002). DNA polymerase gene sequences indicate Western and forest tent caterpillar viruses form a new taxonomic group within baculoviruses. J. Invertebr. Pathol. 81: 131-147.

O'Reilly DR and Miller LK (1989). A baculovirus blocks insect molting by producing ecdysteroid UDP-glucosyl transferase. Science 245: 1110-1112.

Phanis C, Miller D, Cassar S, Tristem M, et al. (1999). Identification and expression of two baculovirus gp37 genes. $J$. Gen. Virol. 80: 1823-1831.

Relly LM and Guarino LA (1996). The viral ubiquitin gene of autographa californica nuclear polyhedrosis virus is not essential for viral replication. Virology 218: 243-247.

Rohrmann G (1986) Polyhedrin structure. J. Gen. Virol. 67: 1499-1513.

Tamura K, Peterson D, Peterson N, Stecher G, et al. (2011). MEGA5: molecular evolutionary genetics analysis using maximum likelihood, evolutionary distance, and maximum parsimony methods. Mol. Biol. Evo. 28: 2731-2739.

Theilmann D, Blissard G, Bonning B, Jehle J, et al. (2005). Baculoviridae. Virus taxonomy: eighth report of the International Committee on Taxonomy of Viruses. Elsevier, San Diego, 177-185.

Zanotto PM, Kessing BD and Maruniak JE (1993). Phylogenetic interrelationships among baculoviruses: evolutionary rates and host associations. J. Invertebr. Pathol. 62: 147-164. 